ELECTRONIC RESEARCH ANNOUNCEMENTS

OF THE AMERICAN MATHEMATICAL SOCIETY

Volume 5, Pages 76-81 (June 28, 1999)

S $1079-6762(99) 00064-5$

\title{
THE FIRST EIGENVALUE OF A RIEMANN SURFACE
}

\author{
ROBERT BROOKS AND ERAN MAKOVER
}

(Communicated by Walter Neumann)

\begin{abstract}
We present a collection of results whose central theme is that the phenomenon of the first eigenvalue of the Laplacian being large is typical for Riemann surfaces. Our main analytic tool is a method for studying how the hyperbolic metric on a Riemann surface behaves under compactification of the surface. We make the notion of picking a Riemann surface at random by modeling this process on the process of picking a random 3-regular graph. With this model, we show that there are positive constants $C_{1}$ and $C_{2}$ independent of the genus, such that with probability at least $C_{1}$, a randomly picked surface has first eigenvalue at least $C_{2}$.
\end{abstract}

In this note, we announce a collection of results $([10,11,12])$ connected to the behavior of the first eigenvalue $\lambda_{1}(S)$ of a compact Riemann surface of large genus, endowed with a metric of constant curvature -1 . These results have as their common theme that the phenomenon of $\lambda_{1}$ large is in some sense typical. To make the notion of "typical" precise, we model the process of picking a Riemann surface at random on the process of picking a 3-regular graph at random.

The idea of studying the first eigenvalue of a Riemann surface via the study of eigenvalues of 3-regular graphs comes from the work of Buser [13, 14]. In effect, our approach here is a variation on his idea, where we first study the behavior of $\lambda_{1}$ on finite-area Riemann surfaces connected to 3-regular graphs, and then see how $\lambda_{1}$ changes when we compactify the surface.

Our main analytic tool is a method for studying how the hyperbolic metric of a finite-area Riemann surface behaves under such a compactification. This method was introduced in [6], and is based on the Ahlfors-Schwarz Lemma ([1]; see also $[5])$.

We then have:

Theorem 1 ([10]). For all $\epsilon$, there exists $N$ such that, for $g \geq N$, there is a compact Riemann surface $S_{g}$ of genus $g$ satisfying

$$
\lambda_{1}\left(S_{g}\right) \geq \frac{171}{784}-\epsilon
$$

The number 171/784 comes from the improvement by Luo, Rudnick, and Sarnak [17] of the Selberg 3/16 Theorem [18]. If Selberg's conjecture were true, we would be able to replace $3 / 16$ by $1 / 4$, the best possible value. More generally, Theorem 1

Received by the editors March 25, 1999.

1991 Mathematics Subject Classification. Primary 58G99.

Partially supported by the Israel Science Foundation, founded by the Israel Academy of Arts and Sciences, the Fund for the Promotion of Research at the Technion, and the New York Metropolitan Fund. 
contains a method to build from a collection of Riemann surfaces $\left\{S_{i}\right\}$ of large first eigenvalue a larger collection of surfaces whose genera may include all but finitely many genera, whose first eigenvalues satisfy a similar bound.

To state our next results, let $\mathcal{F}_{n, k}$ denote the set of $k$-regular graphs on $n$ vertices, and $\mathcal{F}_{n, k}^{*}$ the set of pairs $(\Gamma, \mathcal{O})$, where $\Gamma \in \mathcal{F}_{n, k}$ and $\mathcal{O}$ is an orientation on $\Gamma$ - that is, for each vertex $v$ of $\Gamma, \mathcal{O}$ prescribes a cyclic ordering of the edges emanating from $\mathcal{O}$. Let $\mathcal{F}_{n, k}^{!}$denote the subset of $\mathcal{F}_{n, k}$ consisting of graphs without loops, double edges, or 3 -cycles. As a probability space, $\mathcal{F}_{n, k}^{!}$has positive measure in $\mathcal{F}_{n, k}$ bounded away from 0 as $n \rightarrow \infty$.

We describe a way of associating to the pair $(\Gamma, \mathcal{O}) \in \mathcal{F}_{n, 3}$ a compact Riemann surface $S^{C}(\Gamma, \mathcal{O})$. By a theorem of Belyi [2], the surfaces that arise in this way are dense in the space of all compact Riemann surfaces.

We then have:

Theorem 2 ([11]). There exists a constant $C_{1}$ with the following property:

(a) If $\Gamma$ is picked randomly from $\mathcal{F}_{n, 4}^{!}$for $n$ odd, then with probability $\rightarrow 1$ as $n \rightarrow \infty$, there is an orientation $\mathcal{O}$ on $\Gamma$ so that for any splitting of $(\Gamma, \mathcal{O})$ to a 3-regular graph $\left(\Gamma^{\prime}, \mathcal{O}^{\prime}\right)$, the surface $S^{C}\left(\Gamma^{\prime}, \mathcal{O}^{\prime}\right)$ satisfies:

(i) $S^{C}\left(\Gamma^{\prime}, \mathcal{O}^{\prime}\right)$ has genus $\frac{n+1}{2}$;

(ii) $\lambda_{1}\left(S^{C}\left(\Gamma^{\prime}, \mathcal{O}^{\prime}\right)\right) \geq C_{1}$.

(b) If $\Gamma$ is picked randomly from $\mathcal{F}_{n, 3}^{!}$with $n \equiv 2(\bmod 4)$, then with probability $\rightarrow$ 1 as $n \rightarrow \infty$, there is an orientation $\mathcal{O}$ on $\Gamma$ such that the surface $S^{C}\left(\Gamma^{\prime}, \mathcal{O}^{\prime}\right)$ satisfies:

(i) $S^{C}\left(\Gamma^{\prime}, \mathcal{O}^{\prime}\right)$ has genus $\frac{n+2}{4}$;

(ii) $\lambda_{1}\left(S^{C}\left(\Gamma^{\prime}, \mathcal{O}^{\prime}\right)\right) \geq C_{1}$.

Theorem 3 ([12]). There exist constants $C_{2}, C_{3}, C_{4}$, and $C_{5}$ with the following property: if $(\Gamma, \mathcal{O})$ is picked randomly from $\mathcal{F}_{n, 3}^{*}$, then, as $n \rightarrow \infty$, the surface $S^{C}(\Gamma, \mathcal{O})$ will have the following properties, with probability at least $C_{2}$ :

(a) $\lambda_{1}\left(S^{C}(\Gamma, \mathcal{O})\right) \geq C_{3}$.

(b) The length of the shortest geodesic $\operatorname{syst}\left(S^{C}(\Gamma, \mathcal{O})\right)$ of $S^{C}(\Gamma, \mathcal{O})$ satisfies

$$
\operatorname{syst}\left(S^{C}(\Gamma, \mathcal{O})\right) \geq C_{4}
$$

(c) The diameter $\operatorname{diam}\left(S^{C}(\Gamma, \mathcal{O})\right)$ satisfies

$$
\operatorname{diam}\left(S^{C}(\Gamma, \mathcal{O})\right) \leq C_{5} \log \left(\operatorname{genus}\left(S^{C}(\Gamma, \mathcal{O})\right)\right) .
$$

\section{ACKNOWLEDGEMENTS}

The first author would like to thank the UMPA of the École Normale Supérieure of Lyon, for its hospitality during the time when this paper was written.

\section{Open and Closed Riemann surfaces}

Let $S^{O}$ be an open Riemann surface, carrying a complete metric of constant curvature -1 and finite area. Then there is a unique compactification $S^{C}$ of $S^{O}$ whose conformal structure is uniquely determined from $S^{O}$. In general, $S^{C}$ need not carry a hyperbolic metric, but under favorable circumstances it will, and indeed the hyperbolic metrics on $S^{O}$ and $S^{C}$ will be closely related. 
Theorem 4 ([6]). For all $\epsilon$, there exists $L$ such that, if all the cusps of $S^{O}$ have length $\geq L$, then there are canonically defined cusp neighborhoods $\left\{\mathcal{U}_{i}\right\}$ on $S^{O}$ and $S^{C}$ such that the hyperbolic metrics $d s_{O}^{2}$ and $d s_{C}^{2}$ satisfy

$$
\frac{1}{(1+\epsilon)} d s_{O}^{2} \leq d s_{C}^{2} \leq(1+\epsilon) d s_{O}^{2}
$$

outside the sets $\left\{\mathcal{U}_{i}\right\}$.

See [6] for a precise statement. The length of a cusp is the length of the longest closed horocycle about the cusp.

The inverse process was described in [10]:

Theorem 5 ([10]). Given L, there exisis a number $R$ with the following property: If $S$ is a compact Riemann surface, and $\left\{p_{1}, \ldots, p_{k}\right\}$ points on $S$ such that

(a) The injectivity radius about each point is at least $R$, and

(b) The balls $B\left(p_{i}, R\right)$ of radius $R$ about the points $p_{i}$ are pairwise disjoint, then $S-\left\{p_{1}, \ldots, p_{k}\right\}$ has cusps of length $\geq L$.

Using this, one shows:

Theorem $6([6,10])$. For $L$ sufficiently large, there is a constant $C(L)$ such that:

(i) The Cheeger constants $h\left(S^{O}\right)$ and $h\left(S^{C}\right)$ satisfy:

$$
\frac{1}{C(L)} h\left(S^{O}\right) \leq h\left(S^{C}\right) \leq C(L) h\left(S^{O}\right)
$$

(ii) The first eigenvalues $\lambda_{1}\left(S^{O}\right)$ and $\lambda_{1}\left(S^{C}\right)$ satisfy

$$
\frac{1}{C(L)} \lambda_{1}\left(S^{O}\right) \leq \lambda_{1}\left(S^{C}\right) \leq C(L) \lambda_{1}\left(S^{O}\right)
$$

(iii) The shortest closed geodesics satisfy

$$
\frac{1}{C(L)} \operatorname{syst}\left(S^{O}\right) \leq \operatorname{syst}\left(S^{C}\right) \leq C(L) \operatorname{syst}\left(S^{O}\right) .
$$

Furthermore, $C(L) \rightarrow 1$ as $L \rightarrow \infty$.

By combining this result with the technique of [15] of closing off cusps by forming handles, we prove

Theorem 7 ([10]). Let $\left\{S_{i}\right\}$ be a collection of compact Riemann surfaces with the following properties:

(a) There exists $\lambda>0$ such that $\lambda_{1}\left(S_{i}\right)>\lambda$ for all $i$.

(b) $\operatorname{syst}\left(S_{i}\right) \rightarrow \infty$ as $i \rightarrow \infty$.

(c) For every $C>1$ there exists an $N$ such that

$$
\{x \in \mathbb{R}: x>N\} \subset \bigcup_{i}[1, C]\left(\operatorname{genus}\left(S_{i}\right)\right) .
$$

Then, for all $\epsilon$, there exists $N$ such that for $g \geq N$ there exists a surface $S_{g}$ of genus $g$ with

$$
\lambda_{1}\left(S_{g}\right) \geq \lambda-\epsilon .
$$

Applying this theorem to the compactifications of the modular surfaces then gives Theorem 1 . 


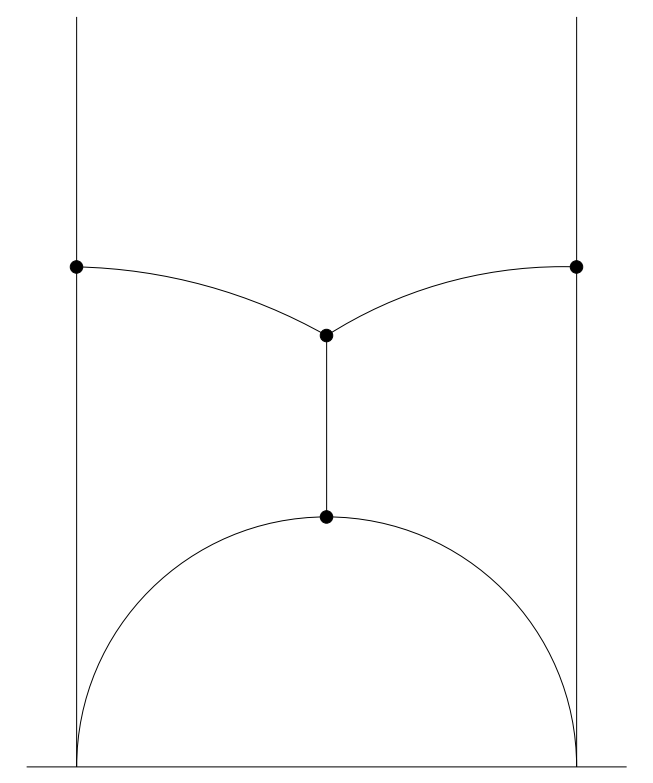

Figure 1. The marked ideal triangle $T$.

\section{RiEMANN SURFACES AND 3-REgUlAR GRAPHS}

Let $\Gamma$ be a 3-regular graph. An orientation $\mathcal{O}$ of $\Gamma$ is an assignment to each vertex $v$ of $\Gamma$ of a cyclic ordering of the edges emanating from $v$. It is clear that a 3 -regular graph on $n$ vertices possesses $2^{n}$ orientations.

To the pair $(\Gamma, \mathcal{O})$ we will assign two surfaces $S^{O}(\Gamma, \mathcal{O})$ and $S^{C}(\Gamma, \mathcal{O})$. The surface $S^{O}(\Gamma, \mathcal{O})$ is obtained by gluing one copy of the ideal hyperbolic triangle $T$ shown in Figure 1 for each vertex of $\Gamma$, such that the natural orientation of the geodesic segments on $T$ matches up with the orientation about the vertex. Whenever two vertices are joined by an edge, we glue the corresponding triangles together, subject to the following conditions:

(i) The geodesic segments on the copies of $T$ are glued together.

(ii) The orientations on the copies of $T$ (as complex manifolds with boundary) are preserved.

The surfaces $S^{C}(\Gamma, \mathcal{O})$ are the compactifications of the surfaces $S^{O}(\Gamma, \mathcal{O})$.

As discussed in [8], the geometry and even the topology of the surfaces $S^{O}(\Gamma, \mathcal{O})$ and $S^{C}(\Gamma, \mathcal{O})$ depend very strongly on the orientation $\mathcal{O}$. We will say that a path $\gamma$ on $(\Gamma, \mathcal{O})$ is a left-hand-turn path if, whenever it arrives at a vertex, it turns left according to the orientation $\mathcal{O}$. Then each cusp of $S^{O}(\Gamma, \mathcal{O})$ is associated to a unique left-hand-turn path. If we denote by $\#(L H T)$ the number of these paths, then the genus of $S^{C}(\Gamma, \mathcal{O})$ is clearly

$$
\operatorname{genus}\left(S^{C}(\Gamma, \mathcal{O})\right)=1+\frac{n-2 \#(L H T)}{4} .
$$

The length of the cusp corresponding to a given left-hand-turn path $\gamma$ is precisely the number of edges in $\gamma$. 
Many geometric properties of the surface $S^{O}(\Gamma, \mathcal{O})$ are reflected in the pair $(\Gamma, \mathcal{O})$. Some of these properties follow from general properties of covering manifolds ([7] and [9]). Other properties depend more delicately on this particular construction. When the graph has no short left-hand-turn paths, then these properties descend to properties on $S^{C}(\Gamma, \mathcal{O})$ via Theorem 6 .

Theorem 8. For some $L$ sufficiently large, there are constants $C_{1}, \ldots, C_{6}$ with the following property: Suppose that $(\Gamma, \mathcal{O})$ has no left-hand-turn paths of length $\leq L$. Then

(i) $C_{1} \lambda_{1}(\Gamma) \leq \lambda_{1}\left(S^{C}(\Gamma, \mathcal{O})\right) \leq C_{2} \lambda_{1}(\Gamma)$.

(ii) $C_{3} h(\Gamma) \leq S^{C}(\Gamma, \mathcal{O}) \leq C_{4} h(\Gamma)$.

(iii) $\log (\operatorname{syst}(\Gamma)) \leq \operatorname{syst}\left(S^{C}(\Gamma, \mathcal{O})\right) \leq C_{5} \operatorname{syst}(\Gamma)$.

(iv) ([11]) $\operatorname{diam}\left(S^{C}(\Gamma, \mathcal{O})\right) \leq C_{6} \operatorname{diam}(\Gamma)$.

With such strong control over the surface $S^{C}(\Gamma, \mathcal{O})$, one might be led to expect that the surfaces $S^{C}(\Gamma, \mathcal{O})$ are rather rare. It is therefore rather surprising that they in fact are quite common.

Theorem 9 ([2, 12]). Given any compact Riemann surface $S$, there are arbitrarily small deformations $S_{\epsilon}$ of $S$ such that $S_{\epsilon}=S^{C}(\Gamma, \mathcal{O})$ for some pair $(\Gamma, \mathcal{O})$.

\section{MODELS OF RANDOM GRAPHS}

Theorems 2 and 3 are now obtained by an analysis of the process of picking a random graph. To carry out this analysis, we make use of the model of random graphs considered by Bollobás [3, 4]. In this model, a $k$-regular graph on $n$ vertices is constructed at random by putting $n k$ balls into a hat, $k$ balls for each vertex. The balls are drawn out of the hat in pairs, and an edge drawn between $v_{1}$ and $v_{2}$ each time a pair of balls corresponding to $v_{1}$ and $v_{2}$ is drawn. An orientation on the graph may be determined by the order in which the corresponding pairs are drawn.

We will need the following results of [3] and [4]:

Theorem 10. (i) ([4]) There is a constant $C_{1}$ such that, as $n \rightarrow \infty$, the probability that $H(\Gamma) \geq C_{1}$ tends to 1 .

(ii) ([3]) Let $X_{1}, \ldots, X_{L}$ denote the random variable

$$
X_{j}=\text { the number of closed paths of length } j \text { in } \Gamma \text {. }
$$

Then, for $L$ fixed and $n \rightarrow \infty$, the variables $X_{1}, \ldots, X_{L}$ tend to independent Poisson distributions.

To establish Theorem 2 (a), we seek the probability that a randomly chosen 4regular graph will have an orientation with precisely one left-hand-turn path. Using ideas of [20,21], it is shown in [11] that this will happen with probability $\rightarrow 1$ as long as $\Gamma$ has no closed loops of length 1 . The proof of Theorem 2 (b) is similar, using [19] in place of [20, 21].

To establish Theorem 3, we estimate the probability that the pair $(\Gamma, \mathcal{O})$ has no left-hand-turn paths of length $\leq L$. This will certainly be the case if it has no closed paths of length $\leq L$ whatsoever, from which Theorem 3 follows. By refining this argument, we may get substantially better estimates for the constant $C_{2}$. 


\section{REFERENCES}

[1] L. Ahlfors, An extension of Schwarz' lemma, Trans. AMS 43 (1938), 359-364.

[2] A. Belyi, On Galois extensions of a maximal cyclotomic polynomial, Math. USSR Izv. 14 (1980), 247-256. MR 80f: 12008

[3] B. Bollobás, Random graphs, Academic Press, 1985. MR 87f:05152

[4] B. Bollobás, The isoperimetric number of random regular graphs, Europ. J. Comb. 9 (1988), 241-244. MR 89e:05180

[5] R. Brooks, Some geometric aspects of the work of Lars Ahlfors, to appear in Proc. Ahlfors Mem. Lect.

[6] R. Brooks, Platonic surfaces, Comm. Math. Helv. 74 (1999), 156-170. CMP 99:09

[7] R. Brooks, The spectral geometry of a tower of coverings, J. Diff. Geom. 23 (1986), 97-107. MR 87j:58095

[8] R. Brooks, Twist surfaces, to appear in Proc. Cortona Conf.

[9] R. Brooks, Some remarks on volume and diameter of Riemannian manifolds, J. Diff. Geom. 27 (1988), 81-86. MR 88m:53074

[10] R. Brooks and E. Makover, Riemann surfaces with large first eigenvalue, to appear.

[11] R. Brooks and E. Makover, The spectral geometry of Belyi surfaces, to appear in Isr. Math. Conf. Proc.

[12] R. Brooks and E. Makover, Random construction of Riemann surfaces, to appear.

[13] P. Buser, Cubic graphs and the first eigenvalue of a Riemann surface, Math. Zeit. 162 (1978), 87-99. MR 80b:58061

[14] P. Buser, On the bipartition of graphs, Discrete Appl. Math. 9 (1984), 105-109. MR 86a:05072

[15] M. Burger, P. Buser, and J. Dodziuk, Riemann surfaces of large genus and large $\lambda_{1}$, in: T. Sunada (ed.), Geometry and Analysis on Manifolds, Springer Lecture Notes 1339 (1988), pp. 54-63. MR 90e:58156

[16] J. Cheeger, A lower bound for the smallest eigenvalue of the Laplacian, in: Gunning (ed.), Problems in Analysis, Princeton University Press (1970), pp. 195-199. MR 53:6645

[17] W. Luo, Z. Rudnick, and P. Sarnak, On Selberg's eigenvalue conjecture, Geom. Funct. Anal. 5 (1995), 387-401. MR 96h:11045

[18] A. Selberg, On the estimation of Fourier coefficients of modular forms, in: A. L. Whiteman (ed.), Theory of Numbers, Proc. Symp. Pure Math. 8 (1965), pp. 1-15. MR 32:93

[19] C. Thomassen, Bidirectional retracting-free double tracings and upper imbeddability, J. Comb. Th. B 50 (1990), 198-207. MR 91j:05041

[20] N. H. Xuong, Sur les immersions d'un graphe dans les surfaces orientables, C. R. Acad. Sci. Paris 283 (1976), 745-747. MR 55:13429

[21] N. H. Xuong, Sur quelques classes des graphes possédant des propriétés remarquables, C. R. Acad. Sci. Paris 283 (1976), 813-816. MR 54:12560

Department of Mathematics, Technion-Israel Institute of Technology, Haifa, IsRAEL

E-mail address: rbrooks@tx.technion.ac.il

Department of Mathematics and Computer Science, Drake University, Des Moines, IA 50311

Current address: Department of Mathematics, Dartmouth College, Hanover, NH

E-mail address: eranm@math.huji.ac.il 